Rev. Elev. Méd. vét. Pays trop., 1972, 25 (3) : 397-408

\title{
Influence des supplémentations minérales sur le croît et sur certains témoins biochimiques du métabolisme minéral chez des bovins tropicaux
}

\author{
par H. CALVET $\left(^{*}\right)$, D. FRIOT $\left(^{*}\right)$ et J. CHAMBON $\left(^{*}\right)$
}

\begin{abstract}
RESUME
Les composés minéraux, phosphate bicalcique, "phosphate aluminocalcique " (polyfos), phosphate disodique, incorporés à une ration de base (50 p. 100 de coque d'arachide mélassée à 20 p. 100,49 p. 100 de farine de maïs, 1 p. 100 d'urée) sont administrés pendant 17 semaines à des taurillons zébus, âgés de 4 à 5 ans, ramenés du milieu naturel et entretenus en stabulation, afin de mettre en évidence les éventuelles particularités de ces animaux face à une supplémentation minérale.

L'étude de l'évolution des gains de poids et les résultats des tests biochimiques (hématocrite, urée, $\mathrm{P}, \mathrm{Ca}, \mathrm{Mg}, \mathrm{Cu}, \mathrm{Zn}, \mathrm{Na}, \mathrm{K}$ ) ont permis de constater:

- l'amélioration du gain de poids par un apport excédentaire de sels minéraux;

- la supériorité du phosphate bicalcique sur le phosphate disodique et sur le phosphate alumino calcique.
\end{abstract}

L'estimation des besoins réels en éléments minéraux, chez les bovins tropicaux constitue un problème encore incomplètement résolu. L'absence de données rigoureuses dans ce domaine s'avère gênante dans de nombreux cas et en particulier quand il s'agit de fixer les quantités et la nature des matières minérales qu'il convient d'incorporer aux rations visant à l'engraissement intensif de ce type de bétail. A des apports trop importants, correspond, en effet, un risque de gaspillage d'éléments dont le prix de revient, au Sénégal, n'est pas négligeable. Par contre, un taux de minéralisation trop faible peut constituer un frein pour la production.

(*) Laboratoire National de l'Elevage et de Recherches Vétérinaires, Dakar-Hann, B.P. no 2057 , Sénégal.
Ces divers problèmes, et en particulier celui d'une éventuelle liaison entre la teneur en éléments minéraux de la ration et le gain de poids, vont être abordés au cours de l'expérimentation qui fait l'objet de cette note. Il s'agit là d'une approche restreinte, au stade du laboratoire, de questions qui seront traitées plus largement sur le terrain, à l'occasion du fonctionnement du centre de prévulgarisation des supplémentations minérales de Labgar $\left({ }^{* *}\right)$

(**) Le centre de prévulgarisation des supplémentations minérales de Labgar est implanté en pleine zone sylvo-pastorale du Sénégal. Sa mise en place et son fonctionnement à partir d'octobre 1971 résultent d'études antérieures qui ont $\mathrm{mis}$ en évidence dans cette région une polycarence en phosphore, calcium, cuivre favorisant l'extension du botulisme. A l'occasion de ce projet, financé par le Fonds d'Aide et de Coopération, plus d'un millier d'animaux recevront pendant deux ans minimum des suppléments minéraux d'origine diverse et on s'efforcera de dégager les conséquences économiques de ces traitements. 
devant intéresser plus d'un millier de bovins pendant deux années consécutives.

\section{MATERIELS ET METHODES}

Les animaux d'expérience sont des zébus entiers de race Gobra, âgés de 3 à 5 ans, élevés dans le milieu naturel suivant les techniques habituelles pratiquées dans les élevages extensifs du Sénégal. Ils sont répartis au hasard dans quatre lots dont les poids moyens en début d'essai s'établissent ainsi :

TABLEAU N ${ }^{\circ}$ I

Poids moyens des lots en début d'essai

\begin{tabular}{|c|c|c|c|c|}
\hline \multirow{4}{*}{$n$} & Témoins & Lot 1 & Lot 2 & Lot 3 \\
\cline { 2 - 5 } & 5 & 5 & 4 & 4 \\
$\mathrm{x}$ & $267 \pm 27$ & $218 \pm 23$ & $269 \pm 52$ & $229 \pm 32$ \\
\hline
\end{tabular}

Conformément aux normes européennes, les besoins minéraux journaliers de ces animaux peuvent être estimés à $15 \mathrm{~g}$ de phosphore et à $18 \mathrm{~g}$ de calcium.

Les quatre lots reçoivent la même ration de base composée de 50 parties de coque d'arachide mélassée à 20 p. 100 , de 49 parties de farine de maïs et d'une partie d'urée.

Les quantités d'aliment distribuées varient en cours d'expérience et permettent de distinguer trois périodes.

Pendant la première, d'une durée de 8 semaines, les animaux reçoivent $8 \mathrm{~kg}$ d'aliment.

Durant la seconde, correspondant aux 5 semaines suivantes, la ration journalière est portée à $10 \mathrm{~kg}$. Au cours de la troisième et dernière période, étendue sur 4 semaines, la ration comporte $12 \mathrm{~kg}$ d'aliment. Les quantités journalières sont servies pour tous les lots en deux repas (matin et soir). Trois sortes de suppléments minéraux sont distribués.

Dans le lot 1, on utilise du phosphate bicalcique-engrais, importé à Dakar, dont la composition chimique exacte en oligo-éléments ne nous a pas encore été communiquée.

Ce phosphate auquel sont adjoints des excipients, est mis sous forme de fins granulés correspondant à la formule suivante :
Phosphate bicalcique . . . 72 p. 100

Sel . . . . . . . 12 p. 100

Maïs . . . . . . 8 p. 100

Son d'arachide . . . . 8 p. 100

Cent grammes de ces granulés contiennent donc approximativement $16,8 \mathrm{~g}$ de calcium et $12,7 \mathrm{~g}$ de phosphore.

Dans le lot 2, le phosphore utilisé est le « polyfos » obtenu après broyage et calcination de phosphate alumino-calco-ferrique du Sénégal, dont la composition chimique comporte les garanties suivantes:

Teneur minérale en $\mathrm{P} . \quad . \quad 15$ p. 100

Teneur moyenne en $\mathrm{Ca}$. . 7,4 p. 100

Teneur maximale en $F$. . 1,1 p. 100

Teneur moyenne en alumine

$$
\left(\mathrm{Al}^{2} \mathrm{O}^{3}\right) \quad 36 \text { p. } 100
$$

Ce phosphate alumino-calco-ferrique présente une teneur intéressante en oligo-éléments.
Cobalt

Cuivre

Fer

Manganèse

Zinc
$130 \mathrm{mg} / \mathrm{kg}$ $60.000 \mathrm{mg} / \mathrm{kg}$ $150 \mathrm{mg} / \mathrm{kg}$ $440 \mathrm{mg} / \mathrm{kg}$
$70 \mathrm{mg} / \mathrm{kg}$

Il est distribué aux animaux sous 1a forme de granulés dont la composition répond à la formule :

Polyfos . . . . . . . . 54

Carbonate de chaux . . . . . . 18

Sel . . . . . . . 8

Maïs . . . . . . . . 8

Son d'arachide . . . . . . . . 8

Cent $\mathrm{g}$ de ces granulés apportent $10,8 \mathrm{~g}$ de calcium et 8,13 de phosphore.

Le lot 3, enfin, reçoit du phosphate disodique qui est mélangé à la ration.

Le protocole initial prévoyait l'administration de phosphate monosodique dans l'eau de boisson, mais ce dernier, introuvable à Dakar a été remplacé par du disodique $\left(12 \quad \mathrm{H}_{2} \mathrm{O}\right)$ difficilement soluble dans l'eau au $\mathrm{pH}$ normal.

Cent $\mathrm{g}$ de ce produit apportent $8,6 \mathrm{~g}$ de phosphore et $12,8 \mathrm{~g}$ de sodium.

Ces suppléments ont été distribués journellement et en quantité constante $(50 \mathrm{~g}$ de granulés bicalciques, $100 \mathrm{~g}$ de granulés polyfos, $100 \mathrm{~g}$ de disodique) durant les deux premières périodes. 
Les quantités en sont doublées au cours de la dernière période.
Les apports minéraux journaliers au cours de l'expérimentation s'établissent comme ciaprès :

TABLEAU $\mathrm{N}^{\circ} \mathrm{II}$

Apports minéraux journaliers au cours des troıs périodes

\begin{tabular}{|c|c|c|c|c|c|c|c|}
\hline \multirow[t]{3}{*}{ lère për1ode } & \multicolumn{7}{|c|}{ R a t i o n $8 \mathrm{~kg}$} \\
\hline & \multicolumn{3}{|c|}{$\mathrm{c}$ a 1 c $1 \mathrm{um}$} & \multicolumn{3}{|c|}{$P h \circ s p o r e$} & \multirow[b]{2}{*}{$\mathrm{Ca} / \mathrm{P}$} \\
\hline & $\begin{array}{l}\text { Ration } \\
\text { de base }\end{array}$ & $\begin{array}{c}\text { Supplément } \\
\text { minéral }\end{array}$ & Total & $\begin{array}{l}\text { Ration } \\
\text { de base }\end{array}$ & $\begin{array}{l}\text { Supplément } \\
\text { minêral }\end{array}$ & Total & \\
\hline Lot tểmoin & 17.76 & 0 & 17,76 & 14,32 & 0 & 14,32 & 1,24 \\
\hline Lot 1 & $"$ & 8,4 & 26,16 & $"$ & 6,35 & 20,67 & 1,26 \\
\hline Lot 2 & $"$ & $10, B$ & 28,56 & $"$ & 8,13 & 22,45 & 1,27 \\
\hline Lot 3 & $"$ & 0 & 17,76 & $"$ & 8,6 & 22,92 & 0,77 \\
\hline \multicolumn{8}{|l|}{ 2e période } \\
\hline Lot témoin & 22,2 & 0 & 22,2 & 17,9 & 0 & 17,9 & 1,24 \\
\hline Lot 1 & $"$ & 8,4 & 30,6 & $"$ & 6,35 & 24,25 & 1,26 \\
\hline Lot 2 & $"$ & 10,8 & 33,0 & $" 1$ & 8,13 & 26,03 & 1,26 \\
\hline Lot 3 & $"$ & 0 & 22,2 & $"$ & 8,6 & 26,5 & 0,83 \\
\hline \multicolumn{8}{|l|}{ 3e période } \\
\hline Lot têmoin & 26,64 & 0 & 26,64 & 21,48 & 0 & 21,48 & 1,24 \\
\hline Lot 1 & $"$ & 16.8 & 43,44 & "I & 12,7 & 34,18 & 1,27 \\
\hline Lot 2 & $"$ & 21,6 & 48,24 & $"$ & 16,26 & 37,74 & 1,27 \\
\hline Lot 3 & $"$ & 0 & 26,64 & $"$ & 17,2 & 38,68 & 0,68 \\
\hline
\end{tabular}

La ration de base que reçoivent les témoins, paraît donc juste suffisante du point de vue Ca et $\mathbf{P}$ au début de l'expérience. Par la suite, son augmentation quantitative assure une large couverture des besoins.

Les rapports $\mathrm{Ca} / \mathrm{P}$ pour les témoins et les deux premiers lots supplémentés sont équivalents et supérieurs à 1 .

La singularité du lot 3 (phosphate disodique) réside dans son rapport $\mathrm{Ca} / \mathrm{P}$ très inférieur à l'unité.

Les animaux sont pesés une fois par semaine, à jeun et dans les mêmes conditions. lls subissent à cette occasion un prélèvement de sang permettant le dosage de l'hématocrite, de l'urée sanguine, du phosphore inorganique, du calcium, du magnésium, du sodium, du potassium, du cuivre et du zinc.

Ces diverses observations et dosages ont conduit aux résultats présentés par la suite.

\section{PRESENTATION DES RESULTATS}

Les résultats intéressent d'abord l'évolution pondérale des lots. Ils portent ensuite sur les dosages biochimiques et la comparaison des lots pour chaque paramètre étudié.

\subsection{Evolution pondérale}

Chez les ruminants une pesée constitue une mesure incertaine du poids réel de l'animal. L'état de plus ou moins grande répletion des importants réservoirs digestifs, au moment de la pesée, introduit, en effet, des fluctuations sensibles autour de la valeur réelle. Il en résulte, à condition que la tendance au gain ou à la perte soit nette, que la droite de régression des poids en fonction du temps semble constituer la meilleure donnée pour comparer des évolutions pondérales, car cette méthode atténue les variations concernant les mesures ponctuelles. 
Ce procédé de calcul a été utilisé pour exploiter les résultats des présents essais.

En début d'expérience s'est produite une perte de poids généralisée due à l'adaptation des animaux au régime et à la stabulation. Pour améliorer la " linéarité » des droites de régression, l'évolution pondérale est étudiée à partir de la $3^{\text {e }}$ pesée, époque où s'arrête, en général, la perte de poids. Les droites de régression sont alors établies sur les poids pondérés à cette date et sur 16 données.

Le tableau $\mathrm{n}^{\circ}$ III donne les équations de ces droites pour chaque individu.

TABLEAU $N^{\circ}$ III

Equation des droites de régression des poids pondérés.

\begin{tabular}{|c|c|c|c|c|c|c|c|}
\hline $\mathrm{N}^{\circ}$ & Témoins & $\mathrm{N}^{\circ}$ & Lat 1 & $N^{\circ}$ & Lot 2 & $\mathrm{~N}^{\circ}$ & Lot 3 \\
\hline 76 & $Y=2,439 x+98,770$ & 63 & $Y=2,807 x+97,135$ & 71 & $Y=2,975 x+99,775$ & 83 & $Y=2,753 x+98,615$ \\
\hline 78 & $Y=0,817 x+99,997$ & 64 & $Y=2,580 x+100,087$ & 75 & $Y=1,677 x+99,428$ & 79 & $Y=2,032 x+96,797$ \\
\hline 74 & $Y=0,430 x+104,712$ & 65 & $Y=2,066 x+99,567$ & 72 & $Y=2,606 x+97,986$ & 80 & $Y=2,368 x+99,483$ \\
\hline 73 & $Y=0,288 x+102,465$ & 66 & $Y=3,733 x+99,190$ & 70 & $Y=1,219 x+98,713$ & 85 & $Y=1,9 x+105,343$ \\
\hline 77 & $Y=2,023 x+95,933$ & 67 & $\mathrm{Y}=2,707 \mathrm{x}+96,760$ & & & & \\
\hline & $\begin{array}{l}\text { nne lot } \\
\mathrm{Y}=1,146 \mathrm{x}+100,6\end{array}$ & $\begin{array}{r}\bar{x} \\
10 t\end{array}$ & $Y=2,757 x+98,51$ & $\begin{array}{r}\bar{x} \\
10 t\end{array}$ & $Y=2,051 x+98,95$ & $\begin{array}{r}\bar{x} \\
10 t\end{array}$ & $Y=2,282 x+98,28$ \\
\hline
\end{tabular}

On constate que le pourcentage des gains de poids calculé s'établit comme suit:

TABLEAU $N^{\circ} \mathrm{IV}$

Pourcentage calculé de gains de poids par lot

\begin{tabular}{|c|c|c|c|}
\hline Témo1ns & Lot 1 & Lot 2 & Lot 3 \\
\hline $17,99 \pm 18,14$ & $41,68 \pm 11,28$ & $31,79 \pm 19,35$ & $34,10 \pm 8,80$ \\
\hline
\end{tabular}

La lot témoin est le plus hétérogène et le lot 3 le plus homogène du point de vue de pourcentages de gains.

La comparaison pondérale des lots est effectuée par analyse de variance et les valeurs de $\mathrm{F}$ obtenues conduisent aux résultats suivants:

Le lot témoin est très significativement différent des 3 lots supplémentés. $F=9,89^{++}$.

Le lot 1 est très significativement différent du lot témoin. $F=9,51++$.

Il n'y a pas de différence significative entre lot 2 et témoin $(F=2,28)$ pas plus qu'entre le lot 3 et témoin $(F=4,28)$.

Statistiquement, le lot 1 n'est pas différent du lot $2(\mathrm{~F}=1,97)$.

\subsection{Résultats biochimiques}

Les prélèvements de sang effectués chaque semaine ont donné lieu à la mesure de l'hématocrite et aux dosages sériques de l'urée, du phosphore inorganique, du calcium, du magnésium, du sodium, du potassium, du zinc et du cuivre.

Le dosage de l'urée sérique utilise la méthode à l'uréase qui fait passer l'urée à l'état d'ammoniac titré par la suite, colorimétriquement, par le réactif de Nessler.

L'analyse du phosphore inorganique a également fait appel à une méthode colorimétrique utilisant le réactif nitro-vanado molybdique (méthode de Misson). La lecture est faite à 420 millimicrons.

Les autres éléments: $\mathrm{Ca}, \mathrm{Mg}, \mathrm{Na}, \mathrm{K}, \mathrm{Cu}$, 
$\mathrm{Zn}$, sont dosés par spectrophotométrie de

Les résultats de ces analyses sont présentés flamme en absorption atomique sur un appareil dans le tableau $n^{\circ} \mathrm{V}$.

Perkin-Elmer modèle 290.

IABLEAU $\mathrm{N}^{\circ} \mathrm{V}$

Résultats généraux des analyses biochimiques

\begin{tabular}{|c|c|c|c|c|c|c|c|}
\hline & Têmoins & Lot & 1 & Lot & 2 & Lot & 3 \\
\hline Hëmatocrite & $\begin{array}{l}n=80 \\
31,5 \pm 1,9\end{array}$ & $\begin{array}{c}\mathrm{n}=78 \\
34,1\end{array}$ & $\pm 1,2$ & $n=64$ & $\pm 1,7$ & $\begin{array}{l}n=61 \\
31,8\end{array}$ & $\pm 2,0$ \\
\hline Urémie & $\begin{array}{l}n=66 \\
0,340 \pm 0,03\end{array}$ & $\begin{array}{l}\mathrm{n}=56 \\
0,300\end{array}$ & $\pm 0,03$ & $\begin{aligned} & \mathrm{n}=55 \\
& 0,320\end{aligned}$ & $\pm \quad 0,02$ & $\begin{array}{r}n=54 \\
0,340\end{array}$ & $\pm 0,03$ \\
\hline Phosphore & $\begin{array}{l}n=79 \\
77,6 \pm 2,8\end{array}$ & $\begin{array}{c}\pi=80 \\
86,1\end{array}$ & $\pm 3,7$ & $\begin{array}{c}n=63 \\
80,0\end{array}$ & $\pm 3,3$ & $\begin{array}{l}n=58 \\
80,2\end{array}$ & $\pm 3,5$ \\
\hline Calcium & $\begin{array}{l}\mathrm{n}=75 \\
88,1 \pm 1,8\end{array}$ & $\begin{array}{c}n=74 \\
88,3\end{array}$ & $\pm 1,9$ & $\begin{array}{c}n=59 \\
89,0\end{array}$ & $\pm 1,3$ & $\begin{array}{l}n=55 \\
89,3\end{array}$ & \pm 1.6 \\
\hline Magnésium & $\begin{array}{l}n=74 \\
20,21 \pm 0,81\end{array}$ & $\begin{array}{r}n=74 \\
21,03\end{array}$ & \pm 1.71 & $\begin{array}{l}\mathrm{n}=59 \\
21,50\end{array}$ & \pm 0.84 & $\begin{array}{l}n=55 \\
21,33\end{array}$ & $\pm 0,99$ \\
\hline Sodium & 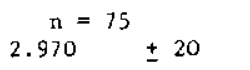 & $\begin{array}{l}n=74 \\
3.001\end{array}$ & \pm 29 & $\begin{array}{l}n=59 \\
2.988\end{array}$ & \pm 21 & $\underset{2.889}{n}=55$ & \\
\hline Potassium & $\begin{array}{l}\mathrm{n}=75 \\
160,7 \pm 3,7\end{array}$ & $\begin{array}{l}\mathrm{n}=73 \\
170,7\end{array}$ & $\pm \quad 4,0$ & $\begin{array}{l}n=58 \\
164,5\end{array}$ & $\pm 3,4$ & $\begin{array}{l}n=54 \\
173,1\end{array}$ & $\pm 6,3$ \\
\hline Zinc & $\begin{array}{l}n=75 \\
0,87 \pm 0,05\end{array}$ & $\begin{array}{r}\mathrm{n}=74 \\
0,90\end{array}$ & $\pm 0,04$ & $\begin{array}{r}n=59 \\
0,94\end{array}$ & $\pm 0,05$ & $\begin{array}{c}\mathrm{n}=56 \\
0.88\end{array}$ & $\pm 0,07$ \\
\hline Cuivre & $\begin{array}{l}n=75 \\
0.62 \pm 0,04\end{array}$ & $\begin{array}{r}\mathrm{n}=59 \\
0,58\end{array}$ & $\pm 0,05$ & $\begin{array}{r}n=59 \\
0,59\end{array}$ & $\pm 0,05$ & $\begin{array}{c}\mathrm{n}=56 \\
0,72\end{array}$ & $\pm 0,06$ \\
\hline
\end{tabular}

Les calculs statistiques par analyse de obtenues pour chaque élément dans chaque lot. variance font intervenir la totalité des données Les conclusions ressortent du tableau suivant,

TABLEAU $\mathrm{N}^{\circ} \mathrm{VI}$

Valeur de F pour les comparaisons des dosages biochimiques

\begin{tabular}{|c|c|c|c|c|c|c|c|c|c|}
\hline Elérıents & \multirow{2}{*}{ Hëmatocrite } & \multirow{2}{*}{ Urémie } & \multirow{2}{*}{$\mathbf{P}$} & \multirow{2}{*}{$\mathrm{Ca}$} & \multirow{2}{*}{$\mathrm{Mg}$} & \multirow{2}{*}{$\mathrm{Na}$} & \multirow{2}{*}{$\mathbf{X}$} & \multirow{2}{*}{$\mathrm{Cu}$} & \multirow{2}{*}{$2 n$} \\
\hline Lots & & & & & & & & & \\
\hline $\begin{array}{l}\text { Sur les } \\
\text { quatre lots }\end{array}$ & $\stackrel{+}{3,34}$ & $\begin{array}{c}0 \\
1,05\end{array}$ & $\begin{array}{l}++ \\
4,64\end{array}$ & $\begin{array}{r}0 \\
<1\end{array}$ & $\begin{array}{l}0 \\
1,33\end{array}$ & $\stackrel{w}{1,29}$ & $\stackrel{++}{7,03}$ & $\begin{array}{c}++ \\
11,5\end{array}$ & $\begin{array}{r}0 \\
<1\end{array}$ \\
\hline $\begin{array}{l}\text { Tëmoins } \\
\qquad \begin{array}{c}\uparrow \\
+ \\
\text { supplémentês }\end{array}\end{array}$ & $\stackrel{+}{4,52}$ & $\begin{array}{c}0 \\
1,67\end{array}$ & $\stackrel{+}{5,98}$ & $\begin{array}{r}0 \\
<1\end{array}$ & $\begin{array}{l}0 \\
3,58\end{array}$ & 2,54 & $\begin{array}{r}++ \\
13,6\end{array}$ & $\begin{array}{r}0 \\
<1\end{array}$ & $\begin{array}{l}0 \\
1,03\end{array}$ \\
\hline $\begin{array}{c}\text { Têmoins } \\
+ \\
\text { Lat }^{\star} \\
\end{array}$ & $\begin{array}{c}+ \\
4,72\end{array}$ & $<1$ & 12 & $<1$ & $\begin{array}{c}0 \\
1,24\end{array}$ & $\begin{array}{c}0 \\
2,97\end{array}$ & $2^{++}$ & $\begin{array}{l}0 \\
2,3\end{array}$ & $\begin{array}{r}0 \\
<1\end{array}$ \\
\hline \begin{tabular}{c} 
Témoins \\
\multirow{i}{*}{} \\
$\downarrow$ \\
Lat
\end{tabular} & $\stackrel{+}{5,90}$ & $<1$ & $\begin{array}{l}0 \\
1.09\end{array}$ & $\begin{array}{r}0 \\
<1\end{array}$ & $\begin{array}{r}+ \\
4.8\end{array}$ & $\begin{array}{c}0 \\
0,66\end{array}$ & $\begin{array}{c}0 \\
2,51\end{array}$ & $\begin{array}{l}0 \\
2,3\end{array}$ & $\begin{array}{l}0 \\
2,58\end{array}$ \\
\hline $\begin{array}{c}\text { Têmoins } \\
\uparrow \\
\begin{array}{c}\uparrow \\
\text { Lot }\end{array}\end{array}$ & $<1^{0}$ & $<1$ & $\begin{array}{c}0 \\
1,1\end{array}$ & $\begin{array}{r}0 \\
<1\end{array}$ & $\begin{array}{l}0 \\
3,22\end{array}$ & ${ }^{0}, 84$ & $13^{++}$ & 14 & $\begin{array}{r}0 \\
<1\end{array}$ \\
\hline $\begin{array}{l}\text { Lot } \\
+ \\
+ \\
\text { Lot }\end{array}$ & & $<1$ & $\begin{array}{c}+ \\
5,33\end{array}$ & & 3,95 & $<1^{0}$ & $\stackrel{+}{5.07}$ & $<1$ & $<1$ \\
\hline
\end{tabular}


présentant les valeurs de $\mathrm{F}$ pour chaque type de comparaison :

- Une différence non significative est marquée par un point;

— Une différence significative à 5 p. 100 par une croix;

- Une différence significative à 1 p. 100 par deux croix.

\subsection{Discussions des résultats}

Elles abordent successivement le problème de l'évolution des poids dans les quatre lots et celui des résultats biochimiques présentés pour chaque élément par un graphique.

Les courbes sont établies en fonction des trois périodes expérimentales et ont la même origine car elles sont "pondérées" sur la moyenne obtenue à partir de tous les animaux au cours du premier dosage effectué avant tout traitement.

\subsubsection{Evolution pondérale}

La supplémentation minérale a produit, dans l'ensemble des lots qui en ont bénéficié et par rapport au lot témoin, un gain de poids supérieur.

L'explication de ce fait peut tenir à une des trois hypothèses suivantes :

1. Il est possible que la ration de base, conçue pour couvrir théoriquement les besoins, n'y soit pas parvenue en raison d'une mauvaise utilisation des matières minérales qu'elle contient.

Les gains de poids insuffisants, enregistrés dans le lot témoin, résulteraient alors d'une carence minérale par défaut d'absorption.

2. On peut envisager que tous les animaux, au moment où ils ont été mis en expérience, se trouvaient dans un état de sub-carence liée à une insuffisance chronique des apports minéraux durant toute leur vie antérieure. Ce fait expliquerait le bénéfice qu'ont tiré les lots supplémentés d'un apport excédentaire en éléments minéraux.

3. La dernière hypothèse, la moins satisfaisante, serait qu'il existe chez les bovins tropicaux et dans les conditions normales, une relation entre gain de poids et niveau de supplémentation minérale.
En réalité, nous pensons que la vérité se situe à mi-chemin des deux premières hypothèses.

En effet, au cours de plusieurs expériences de digestibilité, il est apparu que le phosphore et le calcium contenus dans la coque d'arachide étaient de faible valeur biologique.

D'autre part, les essais d'embouche intensive ont permis de constater que les zébus de 4 ou 5 ans semblent, durant leur vie antérieure, avoir accumulé "un retard de croissance", qu'une alimentation rationnelle leur permet de combler rapidement. Ce "rattrapage " exige sans doute des apports minéraux plus importants que ceux correspondant aux normes admises pour les animaux du même âge.

Les lots traités, ayant reçu des suppléments minéraux différents, le deuxième point à considérer est donc l'étude du comportement pondéral de chaque lot par rapport au lot témoin.

Le graphique $\mathrm{n}^{\circ}$ I présente les droites de régression des poids moyens pondérés en fonction du temps.

Ces droites répondent aux équations suivantes :

$$
\begin{aligned}
& \text { Témoins } \mathrm{y}=1,145 \mathrm{x}+100,60 \\
& \text { Lot } 1 \quad \mathrm{y}=2,757 \mathrm{x}+98,51 \\
& \text { Lot } 2 \mathrm{y}=2,051 \mathrm{x}+98,95 \\
& \text { Lot } 3 \mathrm{y}=2,282 \mathrm{x}+98,27
\end{aligned}
$$

La pente la plus forte est celle du lot 1; viennent ensuite, très proches, celles des lots 2 et 3 et enfin celle du lot témoin.

Le phosphate bicalcique semble donc avoir produit le meilleur effet sur le poids, le polyfos et le phosphate disodique lui étant inférieurs. L'insuffisance numérique des données ne permettant pas de séparer nettement ces deux lots, les calculs statistiques confirment ce résultat. En effet, si on compare par analyse de variance le lot témoin à chacun des lots supplémentés, on obtient respectivement pour L $1, \mathrm{~L} 2, \mathrm{~L}, 3$ les valeurs de F suivantes : 9,58 - 3,90-4,27.

Le lot 1 , recevant le phosphate bicalcique a donc le comportement pondéral qui s'écarte le plus de celui des témoins.

Le phosphate disodique et le polyfos ne se distinguent plus significativement des témoins. 


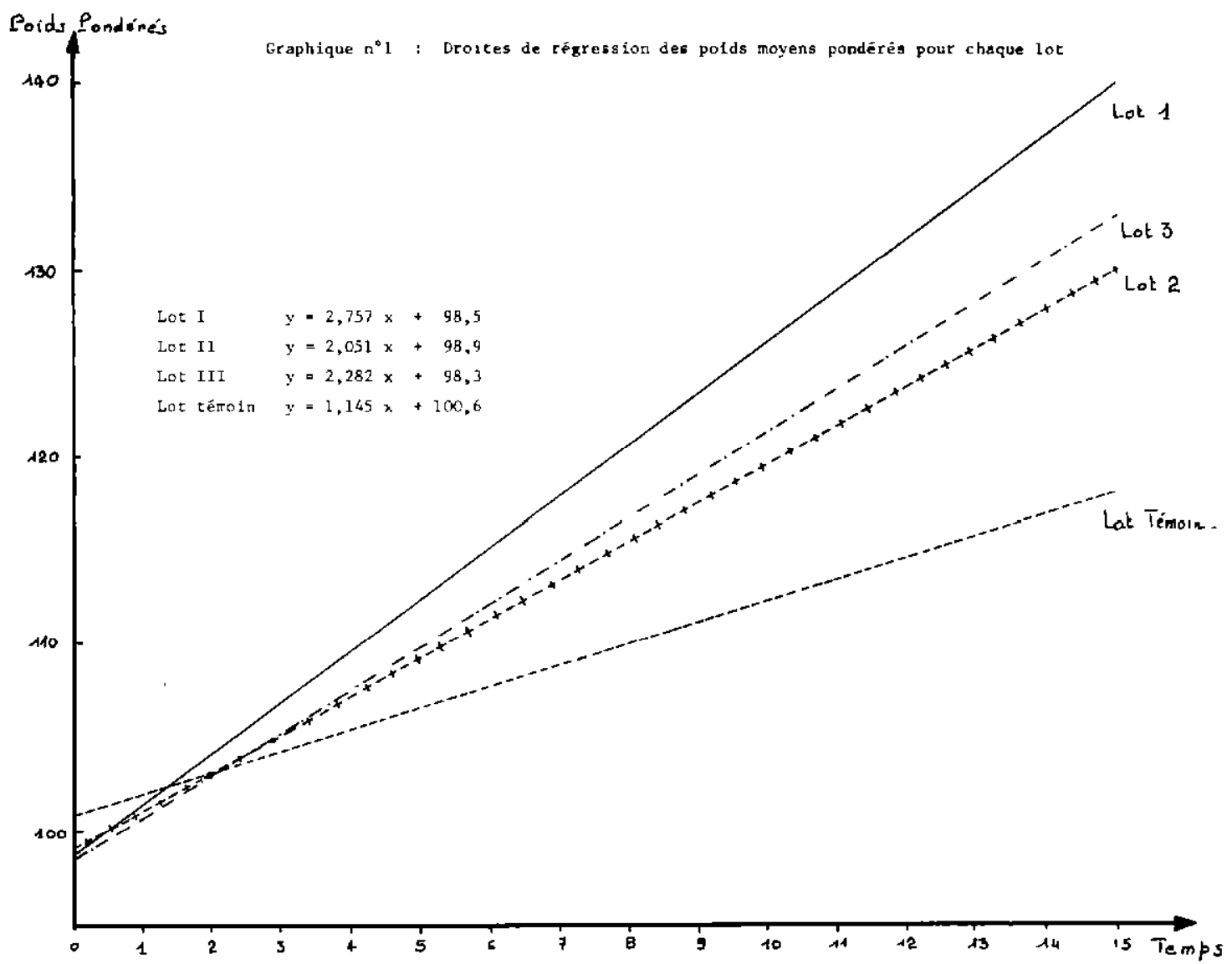

\subsubsection{Résultats biochimiques}

Les valeurs moyennes correspondant aux dosages biochimiques sont présentées dans le tableau IV et les résultats des comparaisons entre lots dans le tableau $\mathrm{V}$.

Les analyses statistiques montrent que la supplémentation minérale a produit un effet sur les hématocrites, la phosphorémie, la kaliémie mais a été sans conséquence pour la magnésémie (sauf dans le lot 2), la natrémie, la cuprémie (sauf le lot 3) et les taux de zinc sérique.

Nous allons envisager séparément chacun de ces éléments.

- Hématocrites. - Les valeurs moyennes des hématocrites sont significativement plus élevées dans les lots traités et parmi ceux-ci, le lot 2 , recevant le polyfos, présente les données les plus distinctes de celles des témoins. Ce fait est probablement la conséquence d'une hyperglobulie provoquée par les apports supplémentaires de fer et de cuivre, éléments pour lesquels le polyfos est particulièrement riche.
- Urémie. - Les taux moyens d'urée sanguine sont comparables dans tous les lots. Un effet de la supplémentation dans ce domaine était peu probable et n'aurait pu résulter que de la présence d'un élément toxique dans les suppléments administrés.

- Eléments minéraux. - Avant d'entreprendre la discussion des résultats, une remarque préalable s'impose.

On admet, en effet, que les valeurs sériques concernant la plupart des éléments minéraux constituent un reflet plus ou moins fidèle des métabolismes correspondants. Il faut cependant souligner la complexité des facteurs qui contribuent à assurer le niveau de la phosphorémie ou de la calcémie par exemple. En effet, les " entrées" en provenance de l'absorption digestive, ou des organes de réserve, interfèrent avec les diverses sorties qui sont l'utilisation, le stockage ou l'élimination. Pour ces diverses raisons, nous parlerons d'un éventuel effet des supplémentations sur le niveau de la calcémie ou de la phosphorémie sans préjuger des 
répercussions sur la totalité du métabolisme calcique ou phosphoré qui ont pu en résulter.

- Phosphorémie. - Sur la totalité des lots, les fluctuations de la phosphorémie ont été beaucoup plus faibles que celles observées généralement. L'entretien en stabulation n'est certainement pas étranger à la stabilisation relative de cette donnée.

Les valeurs moyennes obtenues $(77,6 \pm 2,8$ pour les témoins) sont supérieures à celles observées au cours de deux expérimentations antérieures. Sur un troupeau de Dara, suivi mensuellement, tout au long de l'année 1970, la phosphorémie moyenne a été de $69 \mathrm{mg} / \mathrm{litre}$. Dans les enquêtes effectuées dans la zone sylvo-pastorale du Sénégal, on avait obtenu en 1968 les valeurs de 49,4 $\pm 12,3$ en saison sèche et de $66 \pm 10,9$ en saison des pluies.

Considérés en bloc, les lots supplémentés ont une phosphorémie différente de celle de témoins $(F=5,98)$ mais si l'on compare chaque lot traité avec le lot témoin, on constate que seul le lot 1 accuse une différence hautement significative $\left(F=12^{++}\right)$.

Pour les deux autres, il n'y a pas de différence.

Le phosphate bicalcique est donc le seul supplément qui produise une élévation sensible de la phosphorémie située nettement au-dessus de celle des témoins.

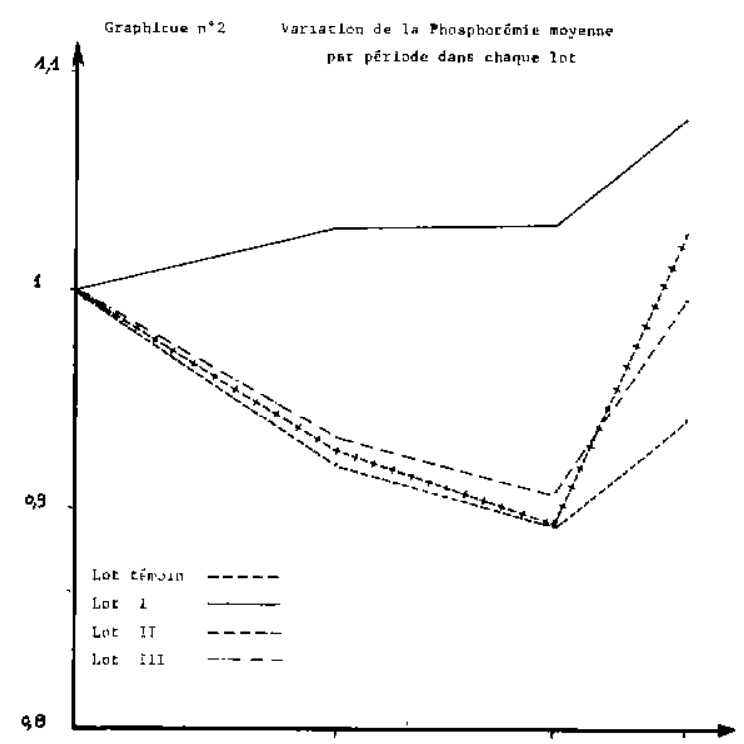

Si l'on considère le graphique $\mathrm{n}^{\mathrm{o}} 2$, on constate que chaque période expérimentale est marquée par un aspect différent des courbes.

Dans le lot 1 , on observe d'abord une élévation de la phosphorémie qui revient en plateau dans la $2^{e}$ période. L'augmentation de la ration entraîne une diminution de concentration du supplément dans l'aliment. En fin d'essai, le fait de doubler les quantités de phosphates se traduit par un brusque relèvement de la courbe. Dans ce lot, l'allure de la phosphorémie paraît liée essentiellement au supplément minéral administré.

Dans les trois autres lots en revanche, on observe, durant la première période, une chute progressive de la phosphorémie malgré les suppléments minéraux distribués. Cette chute se ralentit lorsqu'on augmente la ration et s'inverse totalement en fin d'essai après nouvelle augmentation portant sur la ration et les suppléments.

Pour ces trois derniers lots donc, la phosphorémie paraît beaucoup plus dépendante du phosphore de la ration que de celui apporté par les suppléments.

Ces deux types d'évolution soulignent à nouveau l'efficacité différente sur la phosphorémie du phosphate d'une part et des phosphates disodiques, et alumino-calciques d'autre part.

- Calcémie. - Les taux moyens du calcium sérique ont été relativement constants au cours de l'expérimentation et n'ont pas présenté de différence d'un lot à l'autre.

Le graphique $n^{\circ} 3$ montre une évolution

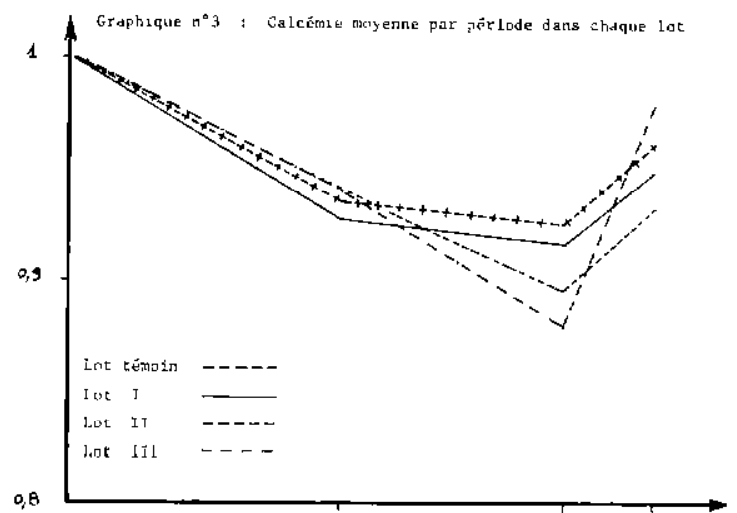


comparable dans tous les lots. Aucune des supplémentations minérales ne semble avoir eu d'effet sur la calcémie.

- Magnésémie. - La magnésémie tout au long de cet essai a paru relativement basse. Les valeurs observées par ailleurs, à Dara et au Ferlo en saison des pluies, sont plus élevées (26,7 mg/litre à Dara et 23,1 au Ferlo). On peut donc penser que la ration de base présente un certain degré de carence en magnésium.

La supplémentation minérale n'a produit une différence significative par rapport aux témoins que dans le lot 2 qui reçoit du polyfos. Ce dernier supplément paraît donc constituer une source de magnésium plus intéressante que les autres phosphates utilisés.

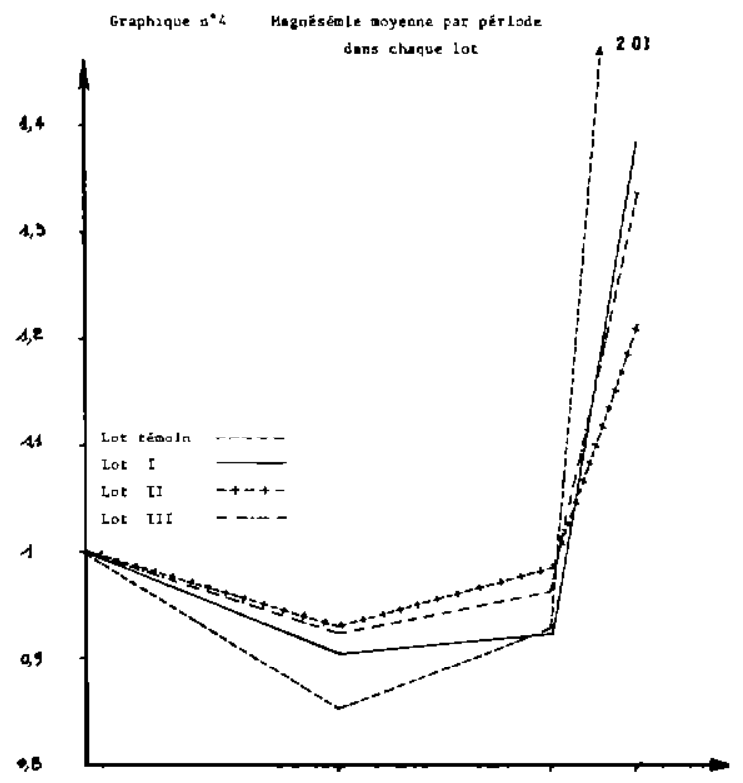

Le graphique $\mathrm{n}^{\circ} 4$ présente ces résultats et souligne la très vive remontée des taux de magnésium dans la dernière partie de l'essai.

Si l'on admet que la ration de base est insuffisante en magnésium, ce fait peut justifier un niveau relativement bas de la calcémie tel qu'il a été observé. On sait, en effet, que calcium et magnésium interfèrent souvent et qu'il s'avère difficile de maintenir une calcémie normale avec des rations carencées en magnésium. Dans les tétanies, l'affaiblissement du taux de magnésium qui précède l'apparition des troubles s'accompagne presque toujours d'une chute de la calcémie.
- Kaliémie. - Les taux moyens de potassium sérique au cours de cet essai ont été inférieurs à ceux rencontrés au Ferlo et en Casamance en saison sèche $(180 \mathrm{mg} / 1$ et $198 \mathrm{mg} / \mathrm{l})$.

Pour cette donnée, on observe des différences hautement significatives entre témoins et supplémentés $(F=13,6)$. Seul, le lot 2 , recevant le polyfos, constitue une exception puisque sa kaliémie moyenne n'est pas différente de celle des témoins.

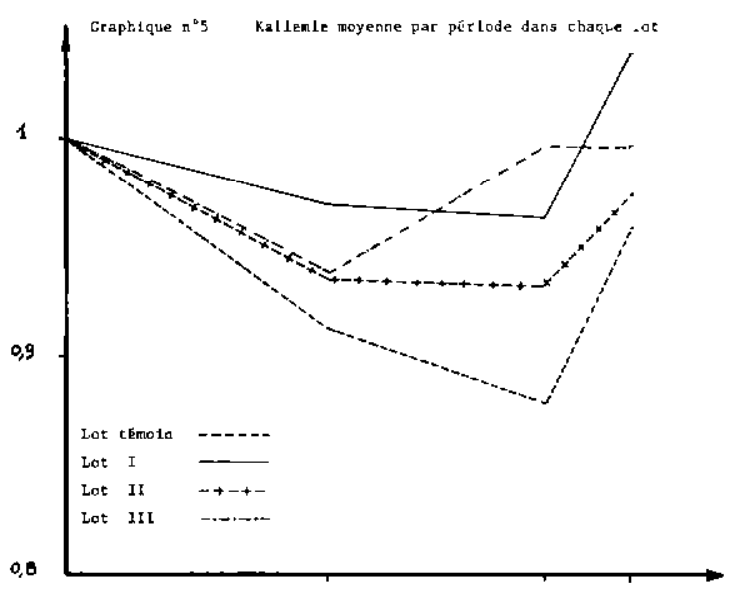

En ce qui concerne le lot 1 , on peut admettre que le bicalcique utilisé contenait sous forme d'impuretés des nitrites de potasses rencontrés fréquemment dans les phosphates naturels et qui ont pu constituer un apport supplémentaire en potassium. La véracité de cette hypothèse sera établie avec le résultat des analyses en cours. Un autre mécanisme peut être invoqué pour justifier la kaliémie élevée dans le lot 3 .

On connaît, en effet, la compétition des ions $\mathrm{K}$ et $\mathrm{Na}$ au niveau de l'élimination urinaire.

Avec le phosphate disodique, on a apporté dans ce lot un excès de sodium qui a pu entraîner une économie de potassium au niveau du tube rénal.

- Natrémie. - Les taux de sodium sériques ne présentent aucun intérêt particulier. Ils sont comparables dans tous les lots, et même dans le $n^{\circ} 3$ dans lequel, malgré un important apport en sodium, on n'observe aucune différence. 
- Cuprémie. - Les taux moyens de cuivre sériques ont été comparables dans tous les lots à l'exception du lot 3 pour lequel on observe une différence hautement significative $(\mathrm{F}=14)$.

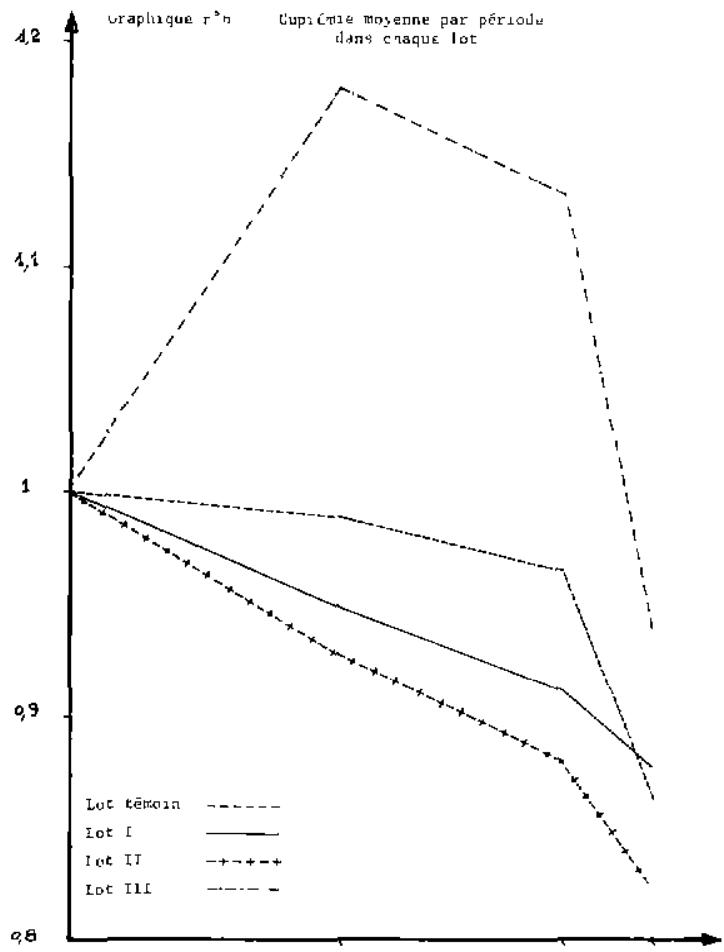

Par rapport aux précédents, le graphique $\mathrm{n}^{\circ} 6$ présente plusieurs singularités:

1. La courbe des témoins est située en position intermédiaire entre le lot recevant le phosphate disodique et les deux autres lots supplémentés.

2. Contrairement à ce qu'on observe pour les autres éléments, la cuprémie chute à la $3^{\text {e }}$ période lorsqu'on augmente la ration. Tout se passe donc comme s'il existait dans la ration un facteur particulier capable de s"opposer à l'absorption du cuivre.

Vis-à-vis de ce facteur antogoniste du cuivre, les suppléments minéraux se comportent de deux façons: Polyfos et bicalcique le potentialisent puisque la cuprémie dans ces lots est inférieure à celle des témoins. Le phosphate disodique semble déprimer cet antagoniste tout au moins dans la première période correspondant à la distribution d'une faible quantité d'aliment. Cet antagoniste du cuivre pourrait être le calcium ou une certaine valeur du rapport $\mathrm{Ca} / \mathrm{P}$.

Dans le cadre restreint de cet essai, on observe, en effet, que lorsque $\mathrm{Ca} / \mathrm{P}$ est supérieur à 1 , la cuprémie est basse. Dans le cas contraire, elle est élevée.

- Zinguémie. - On n'observe pas sur cette donnée de différence d'un lot à l'autre. Les valeurs moyennes en sont inférieures à celles rencontrées en saison sèche au Ferlo et en Casamance $(2,20 \pm 0,5$ et $2,21 \pm 0,36)$.

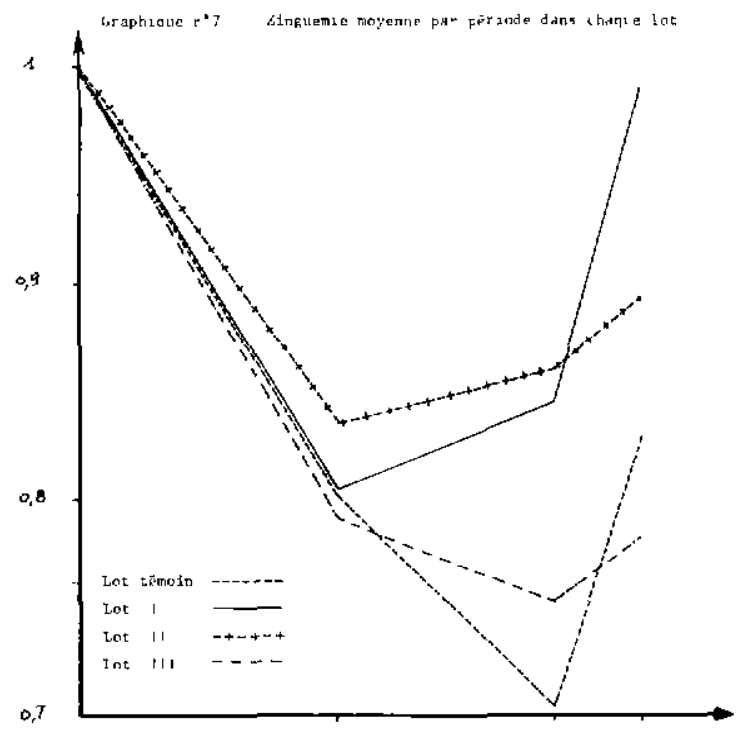

Le graphique $n^{\circ} 7$ présente ces derniers résultats. Les lots supplémentés, en particulier le lot 2, y apparaissent comme étant supérieurs aux lots témoins, ce qui ne confirme pas les calculs.

\section{CONCLUSIONS}

L'objet de cette note était d'aborder les éventuelles particularités des bovins tropicaux face à une supplémentation minérale.

L'expérimentation a fait appel à des taurillons zébus de 4 à 5 ans ramenés du milieu naturel et entretenus en stabulation au laboratoire durant 17 semaines.

La ration distribuée est un mélange de coque d'arachide mélassée à 20 p. 100 et de farine 
de maïs capable de couvrir théoriquement les besoins minéraux des animaux.

L'administration de deux phosphates naturels et d'un phosphate disodique s'est traduite par des apports minéraux largement excédentaires. Dans ces conditions et dans ce cadre expérimental restreint, les résultats ont montré que la supplémentation minérale a produit une amélioration sensible du gain de poids.

Ce fait semble dû d'une part à une faible valeur biologique du calcium et du phosphore apporté par les rations à base de coque d'arachide, mais, surtout à un caractère particulier des zébus utilisés qui, même à l'âge de 4 ans, sont capables de reprendre leur croissance limitée par les privations subies dans les années antérieures. Pour assurer ce "sursaut de croissance " les besoins minéraux se trouvent sensiblement augmentés.

La conséquence de caractère pratique est que les rations utilisées pour l'embouche intensive des zébus de ce type et de cet âge doivent contenir de fortes proportions de sels minéraux, tout spécialement lorsque la coque d'arachide en constitue l'élément de lest.

Parmi les phosphates utilisés, seul le phosphate bicalcique a eu un effet significatif. Le pourcentage de gain de poids observé dans le lot qui le recevait est nettement supérieur à celui des témoins. Ce fait est souligné par les analyses biochimiques dont les résultats montrent pour le lot 1 un taux moyen de phosphate inorganique supérieur à celui observé dans les deux autres et le lot témoin.

\section{SUMMARY}

Influence of mineral feed supplements on growth and some biochemical patterns of mineral metabolism in tropical cattle

Mineral compounds : calcium, phosphate dibasic, "sodjum, phosphate dibasic » (polyfos), incorporated in a basic ration (50 p. cent groundnutshell mixed with $20 \mathrm{p}$. cent molasses, $49 \mathrm{p}$. cent maize flour, and $1 \mathrm{p}$. cent urea) were given for 17 weeks to zebu bulls 4 to 5 years old. The experimental animals were brought from their original bush pasture land and kept in stabulation to demonstrate the effect of the adjunction of a mineral supply.

Te study of the weight gains and the results of biochimical tests (haematocrite, urea, $\mathbf{P}, \mathrm{Ca}, \mathrm{Mg}, \mathrm{Cu}, \mathrm{Zn}, \mathrm{Na}, \mathrm{K}$ ) show:

- the improvement of weight gains when an excess of minerals is given;

- the better biological value of calcium phosphate dibasic compared to sodium phosphate dibasic and polyfos.

\section{RESUMEN}

Influencia de los aditivos minerales sobre el crecimiento y ciertos testigos bioquimicos del metabolismo mineral en los bovinos tropicales

Se administran los compuestos minerales fosfato bicálcico, « fosfato alumino cálcico » (polyfos), fosfato disódico, incorporados en una ración básica (50 p. 100 de cáscara de cacahuete con 20 p. 100 de melaza, 49 p. 100 de harina de maiz, 1 p. 100 de urea), durante 17 semanas, en torillos cebús, de 4 a 5 años de edad, vueltos del medio natural y mantenidos en estabulación para evidenciar las particularidades eventuales de dichos animales para con una ración mineral complementaria.

El estudio de la evolución de los incrementos de peso y los resultados de las pruebas bioquimicas (hematocrito, urea, $\mathrm{P}, \mathrm{Ca}, \mathrm{Mg}, \mathrm{Cu}, \mathrm{Zn}, \mathrm{Na}$, K) permitieron comprobar :

- la mejora del incremento de peso por una adición excedente de sales minerales:

- la superioridad del fosfato bicálcico sobre el fosfato disódico y el fosfato alumino cálcico. 


\section{BIBLIOGRAPHIE}

1. BOHMAN (V. R.), MELENDY (H.) et WADE (M. A.). Influence of dietary supplements on growth of beef calves on semi-desert range. $J$. Anim. Sci., 1961, 20 (3): 553-557.

2. CALVET (H.) et Collab. Aphosphorose et botulisme au Sénégal. Rev. Elev. Méd. vét. Pays trop., 1965, 18 (3) : 249-282.

3. FRIOT (D.) et CALVET ( $\mathrm{H}_{\text {.) }}$. Etude complémentaire sur les carences minérales rencontrées dans les troupeaux du Nord Sénégal. Rev, Elev. Méd. vét. Pays trop., 1971, 24 (3): 393-407.

4. HARRIS (L.E.), BUTCHER (J.E.), JAMES (L.F.) et COOK (C. W.). Influence of protein and phosphorus supplements on range cattle production. $J$. Anim. Sci., 1957, 1059 (Abstract).

5. WARD (H. K.). Supplementation of beef cows grazing on veld, Rhod. J. Agric. Res., 1968, 6 (2) : 93-101. 